\title{
Comparative use of cancer therapies in Australian farm, rural nonfarm and urban residents aged 45 years and older
} \author{
Tony Lowera \\ a Australian Centre for Agricultural Health and Safety, University of Sydney, Dubbo, NSW \\ b National Drug and Alcohol Research Centre, UNSW Sydney, Australia \\ c School of Population Health, University of Western Australia, Perth \\ d Sydney School of Public Health, University of Sydney, NSW, Australia \\ e Corresponding author: julie.depczynski@sydney.edu.au
}

Julie Depczynskia,e, Timothy Dobbins ${ }^{b}$, Bruce Armstrong ${ }^{\mathrm{c}, \mathrm{d}}$ and

\section{Article history}

Publication date: December 2019

Citation: Depczynski J, Dobbins T, Armstrong B, Lower T. Comparative use of cancer therapies in Australian farm, rural nonfarm and urban residents aged 45 years and older. Public Health Res Pract. 2019;29(4):e28341811. First published: 18 October 2018.

https://doi.org/10.17061/phrp28341811

\section{Key points}

- Information on surgical and nonsurgical therapies for prostate, breast and colorectal cancer in farm residents, not previously separated as a group from other rural people, were compared with rural nonfarm and urban counterparts in a large cohort in New South Wales, Australia

- Results suggest that use of surgical therapies was comparable between the three groups

- Farm and rural nonfarm residents were similarly disadvantaged compared with their urban counterparts in the use of some nonsurgical cancer therapies

\section{Abstract}

Objectives and importance of the study: To determine whether use of selected therapies for prostate, breast and colorectal cancer in farm residents differs from that in rural nonfarm and urban counterparts. Little is known about cancer therapies used by farm residents.

Study type: Data linkage cohort study.

Methods: Baseline survey information from the Sax Institute's 45 and Up Study cohort was linked with data from the New South Wales Admitted Patient Data Collection for 2006-2012. Adjusted odds of receiving surgery, chemotherapy, radiotherapy and/or brachytherapy for each cancer were compared between groups, controlling for selected variables.

Results: Differences in the likelihood of surgery for breast and colorectal cancer between groups were not significant. However, for prostate cancer, farm men had $35 \%$ greater odds of prostatectomy than rural nonfarm men (odds ratio [OR] 1.35; 95\% Cl 1.05, 1.72). Urban men were most likely to have had brachytherapy, with three times greater odds of treatment than farm men (OR 2.90; 95\% Cl 1.51, 5.56). Urban women were most likely to have had chemotherapy for breast cancer, having twice the odds of receiving this treatment as farm women (OR $2.24 ; 95 \% \mathrm{Cl} 1.25$, 4.04). The odds of chemotherapy for colorectal cancer among rural nonfarm residents were two-thirds the odds among urban men (OR 0.62; 95\% Cl 0.44, 0.90) and urban women (OR 0.57; $95 \% \mathrm{Cl} 0.37,0.88)$. Age, distance, income and health insurance factors contributed to differences in nonsurgical care between groups.

Conclusions: Cancer-related surgical services for breast and colorectal cancer were comparable between groups. Farm and rural nonfarm residents may have been disadvantaged in relation to nonsurgical therapies for prostate, breast and colorectal cancer compared with urban counterparts. 


\section{Introduction}

Improved survival rates for prostate, breast and colorectal cancers in Australia during the past 30 years have been largely attributed to better diagnostic methods, earlier detection and improvements in cancer treatment. ${ }^{1}$ However, cancer treatments vary in complexity and availability to the general population. Poorer cancer survival in rural areas has been partly attributed to geographic and socio-economic difficulties that rural people experience in accessing optimal cancer treatments. ${ }^{2}$ National data generally show a correlation between decreased expenditure on specialist services and increased remoteness from urban centres. ${ }^{3}$

Cancer risk and treatment indicators are periodically reported by remoteness category, but standard reports do not differentiate between farm residents and other rural people. ${ }^{4}$ Farm residents share unique sociodemographic characteristics as a rural subgroup, described elsewhere. ${ }^{5}$ International reviews suggest that farmer populations have reduced risk of colorectal and perhaps breast cancer, plus possible elevations in prostate cancer. ${ }^{6}$ There is limited information on cancer mortality in Australian farm groups and no published information on how Australian farm residents use cancer treatment services. ${ }^{7-8}$

With a focus on farm residents as the primary group of interest, the study sought to determine whether use of therapies for prostate, breast and colorectal cancer differed between farm, rural nonfarm and urban residents in a large population-based cohort in New South Wales (NSW), Australia. Findings may provide insight into whether farm residents experience more difficulty, or less, in receiving cancer treatment than other rural or urban groups. This has implications for cancer treatment services and assessment of comparative mortality risk.

\section{Methods}

\section{Datasets and linkage}

The study was based on the NSW 45 and Up Study cohort, managed by the Sax Institute in collaboration with major health agency partners. Information from the 45 and Up Study baseline questionnaire was used to define risk factors of interest and categorise participants into farm, rural nonfarm and urban comparison groups. The cohort comprises 267119 NSW residents aged 45 years or older who were enrolled on the database of the Australian Department of Human Services (formerly Medicare Australia) between January 2006 and December 2009. Eligible individuals were randomly sampled from the database, which provides near-complete coverage of the NSW population. Details about 45 and Up Study methods and variables are available elsewhere. ${ }^{9}$

The 45 and Up Study data were linked with corresponding data in the NSW Admitted Patient Data
Collection (APDC) for January 2006 to December 2012 The APDC records all inpatient separations from NSW public and private hospitals, multipurpose services, private day procedure centres and public nursing homes. ${ }^{10}$ Linkage of records between datasets was conducted using probabilistic data linkage methods by the NSW Centre for Health Record Linkage (CHeReL). ${ }^{11}$ $\mathrm{CHeReL}$ also provided geocode-derived distances between the nondisclosed residential address and treating hospital for each record.

\section{Definition of variables}

'Farm residents' were defined as those who indicated that they lived in a 'house on farm' in the 45 and Up Study baseline questionnaire. Rural nonfarm and urban residents were further defined using the Accessibility/ Remoteness Index of Australia (ARIA+), from postcode of residence. ${ }^{4}$ Participants whose ARIA+ classification indicated that they did not live in a 'major city', excluding those who also specified that they lived in a 'house on farm', were defined as rural nonfarm residents. The remaining urban participants were those who lived in a 'major city'. Where ARIA+ was not recorded, allocation was made by cross-checking with postcode of residence and distance to treating hospitals.

Variables of interest considered for inclusion in logistic regression models were age, distance from treating hospital, 'private health insurance with extras' status, and annual household income of $\$ 70000$ or more. The latter approximated levels above or below the NSW 2006 average annual household income of $\$ 70000 .{ }^{12}$ Private health insurance with extras status referred to those with more than basic private hospital coverage, such as for complementary outpatient services used as part of a cancer treatment regime (e.g. ambulance, accommodation services). For distance, the average distance from home address to a treating hospital in all records for each person was used. Parameter estimates for distance to hospital are presented per $10 \mathrm{~km}$ to assist with interpretation. Cancer status was established where a prostate, breast or colorectal cancer diagnosis was recorded in any of the 55 diagnosis code fields of any APDC admission record during the period. Therapeutic procedures were identified within any one of 51 procedure code fields on the APDC record. Diagnosis and procedure codes are based on the International Statistical Classification of Diseases and Related Health Problems, tenth revision, Australian modification (ICD10-AM) and Medicare Benefits Schedule classification systems. ${ }^{10}$

Therapeutic procedures for each selected cancer were categorised into surgical, chemotherapy, radiotherapy and brachytherapy groups. Surgical procedures for women with breast cancer were defined separately for mastectomies (procedure codes 3151800-31524-00) and excision of breast lesions (3150000-31515-00). Surgical procedures for men with 
prostate cancer were defined by radical or laparoscopic prostatectomy (37209-00-37211-09). Surgeries for colorectal cancer for both men and women were defined by procedures relating to colectomies, resections or excision of colorectal tumours (32000-00-32069-00, 32099-00-32108-00). Transurethral resections of prostate, colonoscopies and aspirational biopsies were excluded, to avoid confusion with diagnostic procedures.

Brachytherapy implantation procedures (1532700-15338-00) were compared for prostate cancer cases; and radiotherapy procedures (15100-00-15269-09) were compared for those with a prostate, breast or colorectal cancer admission. Chemotherapy was limited to the administration of antineoplastic pharmacological agents only $(96199-00,96200-00,96201-00,96202-00,96203-$ 00, 96204-00, 96205-00, 96206-00, 96207-00, 96208-00, 96209-00). Sensitivity analyses used wider categories of chemotherapy, such as administration of steroids, antiinfective agents or nutritional pharmacological therapies (96199-00-96209-09).

\section{Analysis}

Analysis was conducted using SAS (Cary, NC: SAS Institute; Version 9.3). Frequencies and average distances from home to hospital were calculated for farm, rural nonfarm and urban residents for specified cancer treatments. Binary logistic regression was used to calculate adjusted odds ratios for selected treatments for each resident group. Variables with $p$ values $<0.25$ on univariate analyses were included in the base model. Effect estimates and standard errors were compared between univariate and multivariable models, and variance inflation factors were calculated to ensure that collinearity did not exist between variables. Interactions between resident group and other factors were created and tested to assess effect modification. Models were progressively reduced using a backward elimination approach (0.05 level), removing nonsignificant variables and accounting for any nonsignificant confounders. Residual plots were examined to identify cases with undue influence in regression models.

Adjusted odds ratios were generated, focusing on farm residents as the referent group. However, differences between rural nonfarm and urban groups were also examined using an urban referent; these are presented where results were significantly different in final models. The difference between groups in the final adjusted odds ratio represents a difference not explained by factors left in the final model, but caused by the effect of group residence or unknown factors.

\section{Results}

Table 1 presents information on variables selected for inclusion in models for the three resident groups within the 45 and Up Study cohort. Tables 2 and 3 present the proportions of farm, rural nonfarm and urban residents who underwent particular cancer therapies for each cancer type, their distance from the treating centre, and the adjusted odds for undergoing therapy. Data for radiotherapy, where at least one cell had less than five cases, are not presented.

\section{Cancer therapies in men}

Farm men were significantly more likely to have a prostatectomy than rural nonfarm men, with 35\% higher odds of the procedure. The average distance to a treating health service was not a significant factor in the likelihood of having a prostatectomy. Small case numbers in the referent group affected calculation of odds ratios for both radiotherapy and chemotherapy for prostate cancer, resulting in wide confidence intervals that included the null value. As such, there was no apparent difference in the adjusted odds of chemotherapy or radiotherapy for prostate cancer. In contrast, urban men were significantly more likely to have brachytherapy, with almost three times the odds of farm men and twice the odds of rural nonfarm men.

Surgical procedures for colorectal cancer did not differ between farm, rural nonfarm and urban men. Urban men had significantly higher odds of undergoing chemotherapy for colorectal cancer than rural nonfarm men. Results of sensitivity analyses for prostate and colorectal cancer chemotherapy supported the main findings.

\section{Cancer therapies in women}

There was little difference between residence groups for surgical procedures of the breast. Distance to a treating health service was not associated with having surgery in final models. Urban women were significantly more likely to undergo chemotherapy for breast cancer than either of the other groups, having 2.2 times the odds of this therapy as farm women. There was little difference between farm, rural nonfarm and urban women in the odds of colorectal surgery. Again, the average distance to a treating hospital was not a significant factor in the likelihood of surgery. As for men, urban women were significantly more likely to undergo chemotherapy for colorectal cancer than rural nonfarm women. Sensitivity analyses for breast and colorectal cancer chemotherapy in women supported the main findings. 
Table 1. Selected characteristics of farm, rural nonfarm and urban residents in the 45 and Up Study cohort, considered for models

\begin{tabular}{|c|c|c|c|c|c|c|c|c|}
\hline \multirow[b]{2}{*}{ Characteristic } & \multicolumn{4}{|c|}{ Men } & \multicolumn{4}{|c|}{ Women } \\
\hline & Total & Farm & $\begin{array}{c}\text { Rural } \\
\text { nonfarm }\end{array}$ & Urban & Total & Farm & $\begin{array}{c}\text { Rural } \\
\text { nonfarm }\end{array}$ & Urban \\
\hline Average age $(n, 95 \% \mathrm{Cl})$ & 123882 & $\begin{array}{c}61.2 \\
(61.0,61.4)\end{array}$ & $\begin{array}{c}63.7 \\
(63.6,63.8)\end{array}$ & $\begin{array}{c}64.3 \\
(64.2,64.4)\end{array}$ & 143192 & $\begin{array}{c}58.6 \\
(58.5,58.8)\end{array}$ & $\begin{array}{c}61.8 \\
(61.7,61.8)\end{array}$ & $\begin{array}{c}62.5 \\
(62.4,62.5)\end{array}$ \\
\hline $\begin{array}{l}\text { Annual income more than } \\
\$ 70000(n)\end{array}$ & $103830^{a}$ & 7813 & 48331 & 47686 & $105384^{b}$ & 7722 & 51790 & 45872 \\
\hline $\begin{array}{l}n(\%)^{c} \text { of those who } \\
\text { responded 'Yes' }\end{array}$ & $\begin{array}{c}34334 \\
(33.1)\end{array}$ & $\begin{array}{l}2313 \\
(29.6)\end{array}$ & $\begin{array}{l}12697 \\
(26.3)\end{array}$ & $\begin{array}{l}19324 \\
(40.5)\end{array}$ & $\begin{array}{l}28477 \\
(27.0)\end{array}$ & $\begin{array}{l}2164 \\
(28.0)\end{array}$ & $\begin{array}{l}10995 \\
(21.2)\end{array}$ & $\begin{array}{l}15318 \\
(33.4)\end{array}$ \\
\hline $\begin{array}{l}\text { Private health insurance } \\
\text { with extras }(n)\end{array}$ & 123907 & 9281 & 57341 & 57285 & 143206 & 10551 & 69467 & 63188 \\
\hline $\begin{array}{l}n(\%)^{c} \text { of those who } \\
\text { responded 'Yes' }\end{array}$ & $\begin{array}{c}60499 \\
(48.8)\end{array}$ & $\begin{array}{l}4339 \\
(46.8)\end{array}$ & $\begin{array}{r}24595 \\
(42.9)\end{array}$ & $\begin{array}{c}31565 \\
(55.1)\end{array}$ & $\begin{array}{l}70454 \\
(49.2)\end{array}$ & $\begin{array}{l}5228 \\
(49.6)\end{array}$ & $\begin{array}{l}29688 \\
(42.7)\end{array}$ & $\begin{array}{l}35538 \\
(56.2)\end{array}$ \\
\hline
\end{tabular}

$\mathrm{Cl}=$ confidence interval

a $16 \%$ of data missing

b $26 \%$ of data missing

c Chi-square test of proportions between resident groups significant to 0.05 level

Table 2. Procedures performed on farm, rural nonfarm and urban men who had a prostate or colorectal cancer diagnosis on any APDC admission record, 2006-2012

\begin{tabular}{|c|c|c|c|c|c|}
\hline Procedures & Total & Farm & $\begin{array}{c}\text { Rural } \\
\text { nonfarm }\end{array}$ & Urban & $\begin{array}{l}\text { Factors remaining in } \\
\text { adjusted model }\end{array}$ \\
\hline Prostate cancer $(n)$ & 5637 & 371 & 2618 & 2648 & \\
\hline \multicolumn{6}{|l|}{$\begin{array}{l}\text { Prostatectomy: radical or laparoscopic } \\
\text { (procedure codes 37209-00-37211-09) }\end{array}$} \\
\hline $\begin{array}{l}n(\%)^{\mathrm{a}} \text { of men with a diagnosis who had a } \\
\text { procedure }\end{array}$ & $\begin{array}{l}2022 \\
(35.9)\end{array}$ & $170(45.8)$ & $901(34.4)$ & $951(35.9)$ & \multirow{3}{*}{$\begin{array}{l}\text { - Age: OR } 0.88 \text { (95\% Cl } 0.87 \text {, } \\
\text { 0.89) } \\
\text { - } \text { Private health insurance with } \\
\text { extras (yes vs no): OR } 1.17 \\
(95 \% \mathrm{Cl} 1.03,1.33)\end{array}$} \\
\hline $\begin{array}{l}\text { Average distance }(\mathrm{km})(95 \% \mathrm{Cl}) \text { from } \\
\text { residence to health service for a first } \\
\text { procedure }\end{array}$ & 2019 & $\begin{array}{r}142.1 \\
(122.4 \\
161.8)\end{array}$ & $\begin{array}{l}93.1 \\
(85.9 \\
100.4)\end{array}$ & $\begin{array}{r}14.1 \\
(12.8 \\
15.5)\end{array}$ & \\
\hline Adjusted OR $(95 \% \mathrm{Cl})$ for procedure & 5634 & 1.00 & $\begin{array}{l}0.74 \\
(0.58 \\
0.95)\end{array}$ & $\begin{array}{r}0.83 \\
(0.64 \\
1.06)\end{array}$ & \\
\hline
\end{tabular}


Table 2. Procedures performed on farm, rural nonfarm and urban men who had a prostate or colorectal cancer diagnosis on any APDC admission record, 2006-2012 (continued)

\begin{tabular}{|c|c|c|c|c|c|}
\hline Procedures & Total & Farm & $\begin{array}{c}\text { Rural } \\
\text { nonfarm }\end{array}$ & Urban & $\begin{array}{l}\text { Factors remaining in } \\
\text { adjusted model }\end{array}$ \\
\hline \multicolumn{6}{|l|}{$\begin{array}{l}\text { Chemotherapy: antineoplastic agents only } \\
\text { (procedure codes 96199-00, 96200-00, } \\
96201-00,96202-00,96203-00,96204-00 \text {, } \\
96205-00,96206-00,96207-00,96208-00 \text {, } \\
\text { 96209-00) }\end{array}$} \\
\hline $\begin{array}{l}n(\%)^{a} \text { of men with a diagnosis who had a } \\
\text { procedure }\end{array}$ & $205(3.6)$ & $8(2.2)$ & $78(3.0)$ & $119(4.5)$ & \multirow{3}{*}{$\begin{array}{l}\text { - Age: OR } 1.04(95 \% \mathrm{Cl} 1.03 \text {, } \\
\text { 1.06) } \\
\text { - } \quad \text { Private health insurance with } \\
\text { extras (yes vs no): OR } 1.56 \\
\text { (95\% Cl 1.15, 2.10) } \\
\text { - } \quad \text { Average distance to health } \\
\text { service (per } 10 \mathrm{~km} \text { ) was a } \\
\text { nonsignificant confounder; } \\
\text { OR }=0.96(95 \% \mathrm{Cl} 0.93,1.00)]\end{array}$} \\
\hline $\begin{array}{l}\text { Average distance }(\mathrm{km})(95 \% \mathrm{Cl}) \text { from } \\
\text { residence to health service for a first } \\
\text { procedure }\end{array}$ & 164 & $\begin{array}{c}56.1 \\
(22.0 \\
90.1)\end{array}$ & $\begin{array}{r}49.0 \\
(30.0 \\
68.0)\end{array}$ & $\begin{array}{c}11.0 \\
(8.0,14.1)\end{array}$ & \\
\hline Adjusted OR (95\% Cl) for procedure & 5600 & 1.00 & $\begin{array}{c}1.14 \\
(0.54 \\
2.42)\end{array}$ & $\begin{array}{l}1.33 \\
(0.62 \\
2.88)\end{array}$ & \\
\hline
\end{tabular}

\section{Radiotherapy (procedure codes} 15100-00-15269-09)

$n(\%)^{\text {a }}$ of men with a diagnosis who had a procedure

- Age: OR $1.06(95 \% \mathrm{Cl} 1.04$, 1.08)

Average distance $(\mathrm{km})(95 \% \mathrm{Cl})$ from residence to health service for a first procedure

198.6 (82.3, 314.8)

128.8

(86.8, 170.8)

- Private health insurance with extras (yes vs no): OR 0.61 (95\% Cl 0.40, 0.92)

- Annual household income more than $\$ 70000$ (yes vs

Adjusted OR (95\% Cl) for procedure $4672 \quad 1.00$

\subsection{1}

(0.24, (0.45, confounder; OR 0.90 $(95 \%$ Cl 0.51, 1.60)]

Brachytherapy: implantations (procedure codes 15327-00-15338-00)

$n(\%)^{a}$ of men with a cancer diagnosis who had a procedure

Average distance $(\mathrm{km})(95 \% \mathrm{Cl})$ from residence to health service for a first procedure

$311(5.5 \%) \quad 18(4.9 \%) \quad 108(4.1 \%) \quad 185(7.0 \%)$

- Annual household income more than $\$ 70000$ (yes vs no): OR $1.38(95 \% \mathrm{Cl} 1.01,1.77)$

$\begin{array}{rrrr}270.6 & 183.7 & 20.4(16.0, & \text { - }\end{array} \quad$ Private health insurance with $\begin{array}{lll}(182.0, & (160.4, & 24.9)\end{array}$ 359.2) 206.9) (95\% Cl 1.36, 2.45)

- Average distance to health service (per $10 \mathrm{~km}$ ): OR 1.06

Adjusted OR (95\% Cl) for procedure $4641 \quad 1.00$

$1.34(0.72, \quad \mathbf{2 . 9 0}(\mathbf{1 . 5 1}$ 2.25)
$(95 \%$ Cl 1.04, 1.08)

- Cohort (rural nonfarm vs urban): OR $0.46(95 \% \mathrm{Cl} 0.34$, 0.63)

\begin{tabular}{|c|c|c|c|c|c|}
\hline Colorectal cancer $(n)$ & 1878 & 96 & 917 & 865 & \\
\hline \multicolumn{6}{|l|}{$\begin{array}{l}\text { Colorectal surgery colectomies, resections, } \\
\text { excisions (procedure codes } 32000-00- \\
32069-00,32099-00-32108-00 \text { ) }\end{array}$} \\
\hline $\begin{array}{l}n(\%)^{a} \text { of men with diagnosis who had a } \\
\text { procedure }\end{array}$ & $\begin{array}{l}1382 \\
(73.6)\end{array}$ & $76(79.2)$ & $664(72.4)$ & $642(74.2)$ & $\begin{array}{l}\text { - Age: OR } 0.99(95 \% \mathrm{Cl} 0.98 \text {, } \\
\text { 1.00) }\end{array}$ \\
\hline $\begin{array}{l}\text { Average distance }(\mathrm{km})(95 \% \mathrm{Cl}) \text { from } \\
\text { residence to health service for a first } \\
\text { procedure }\end{array}$ & 1367 & $\begin{array}{l}78.1(59.0 \\
97.1)\end{array}$ & $\begin{array}{l}54.0(47.5 \\
\quad 60.4)\end{array}$ & $\begin{array}{l}9.6(8.2 \\
11.0)\end{array}$ & \\
\hline Adjusted OR (95\% Cl) for procedure & 1878 & 1.00 & $\begin{array}{l}0.71(0.42 \\
\quad 1.18)\end{array}$ & $\begin{array}{l}0.79(0.47 \\
1.32)\end{array}$ & \\
\hline
\end{tabular}


Table 2. Procedures performed on farm, rural nonfarm and urban men who had a prostate or colorectal cancer diagnosis on any APDC admission record, 2006-2012 (continued)

\begin{tabular}{llll}
\hline Procedures & Total $\quad$ Farm & $\begin{array}{c}\text { Rural } \\
\text { nonfarm }\end{array}$ Urban & $\begin{array}{l}\text { Factors remaining in } \\
\text { adjusted model }\end{array}$ \\
\hline
\end{tabular}

Chemotherapy: antineoplastic agents only (procedure codes 96199-00, 96200-00, 96201-00, 96202-00, 96203-00, 96204-00, 96205-00, 96206-00, 96207-00, 96208-00, 96209-00)

$n(\%)^{a}$ of men with a cancer diagnosis who had a procedure

$\begin{array}{cccc}240 & 9(9.4 \%) & 86(9.4 \%) & 145 \\ (12.8 \%) & & & (16.8 \%) \\ & & & \\ 221 & 64.2(0, & 48.4(30.4, & 9.6(7.8 \\ & 156.5) & 66.5) & 11.4)\end{array}$

- Age: OR 0.98 $(95 \%$ Cl 0.96, 0.995)

Average distance $(\mathrm{km})(95 \% \mathrm{Cl})$ from residence to health service for a first procedure

- Private health insurance with extras (yes vs no): OR 2.97 (95\% Cl 2.11, 4.17)

- Annual household income more than $\$ 70000$ (yes vs no) was a nonsignificant confounder; OR 1.30 $(95 \% \mathrm{Cl} 0.89,1.90)]$

- [Average distance to health Adjusted OR $(95 \% \mathrm{Cl})$ for procedure

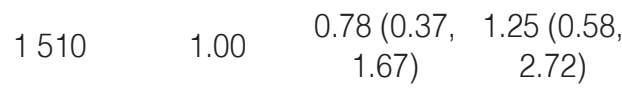
service (per $10 \mathrm{~km}$ ) was a nonsignificant confounder; OR $0.97(95 \%$ Cl 0.93, 1.02)

- Cohort (rural nonfarm vs urban): OR $0.62(95 \% \mathrm{CI}$ $0.44,0.90$ )

APDC = Admitted Patient Data Collection; $\mathrm{Cl}=$ confidence interval; $\mathrm{OR}=$ odds ratio Bold text $=$ odds ratio significant compared with referent resident group to 0.05 level

a Chi-square test of proportions between resident groups significant to 0.05 level

Table 3. Procedures performed on farm, rural nonfarm and urban women who had a breast or colorectal cancer diagnosis on any APDC admission record, 2006-2012

\begin{tabular}{|c|c|c|c|c|c|}
\hline Procedures & Total & Farm & $\begin{array}{c}\text { Rural } \\
\text { nonfarm }\end{array}$ & Urban & Factors remaining in adjusted model \\
\hline Breast cancer & 3188 & 180 & 1514 & 1494 & \\
\hline \multicolumn{6}{|l|}{$\begin{array}{l}\text { Mastectomy (procedure codes } \\
31518-00-31524-00 \text { ) }\end{array}$} \\
\hline $\begin{array}{l}n(\%)^{a} \text { of women with diagnosis who } \\
\text { had procedure }\end{array}$ & $\begin{array}{l}1135 \\
(35.6)\end{array}$ & $\begin{array}{c}63 \\
(35.0)\end{array}$ & $\begin{array}{c}542 \\
(35.8)\end{array}$ & $\begin{array}{c}530 \\
(35.5)\end{array}$ & - Age: OR $1.01(95 \% \mathrm{Cl} 1.00,1.01)$ \\
\hline $\begin{array}{l}\text { Average distance }(\mathrm{km})(95 \% \mathrm{Cl}) \text { from } \\
\text { residence to health service for first } \\
\text { procedure }\end{array}$ & 1117 & $\begin{array}{r}98.4 \\
(67.8 \\
128.9)\end{array}$ & $\begin{array}{c}53.3 \\
(46.2,60.3)\end{array}$ & $\begin{array}{c}11.4 \\
(9.6,13.2)\end{array}$ & \\
\hline Adjusted OR (95\% Cl) for procedure & 3188 & 1.00 & $\begin{array}{c}1.01 \\
(0.73,1.39)\end{array}$ & $\begin{array}{c}0.99 \\
(0.72,1.37)\end{array}$ & \\
\hline
\end{tabular}


Table 3. Procedures performed on farm, rural nonfarm and urban women who had a breast or colorectal cancer diagnosis on any APDC admission record, 2006-2012 (continued)

\begin{tabular}{|c|c|c|c|c|c|}
\hline Procedures & Total & Farm & $\begin{array}{c}\text { Rural } \\
\text { nonfarm }\end{array}$ & Urban & Factors remaining in adjusted model \\
\hline \multicolumn{6}{|l|}{$\begin{array}{l}\text { Excision, re-excision or open biopsy } \\
\text { of lesion (procedure codes } \\
31500-00-31515-00 \text { ) }\end{array}$} \\
\hline $\begin{array}{l}n(\%)^{\mathrm{a}} \text { of women with diagnosis who } \\
\text { had procedure }\end{array}$ & $\begin{array}{l}1915 \\
(60.1)\end{array}$ & $\begin{array}{c}112 \\
(62.2)\end{array}$ & $\begin{array}{c}910 \\
(60.1)\end{array}$ & $\begin{array}{c}893 \\
(59.8)\end{array}$ & \multirow{3}{*}{$\begin{array}{l}\text { - Age: OR } 0.98(95 \% \mathrm{Cl} 0.97,0.99) \\
\text { - Annual household income more } \\
\text { than } \$ 70000 \text { (yes vs no): OR } 1.29 \\
(95 \% \mathrm{Cl} 1.04,1.60)\end{array}$} \\
\hline $\begin{array}{l}\text { Average distance }(\mathrm{km})(95 \% \mathrm{Cl}) \text { from } \\
\text { residence to health service for first } \\
\text { procedure }\end{array}$ & 1842 & $\begin{array}{l}96.7 \\
(76.9 \\
116.4)\end{array}$ & $\begin{array}{c}51.0 \\
(46.0,56.1)\end{array}$ & $\begin{array}{c}11.2 \\
(9.6,12.9)\end{array}$ & \\
\hline Adjusted OR (95\% Cl) for procedure & 2343 & 1.00 & $\begin{array}{c}1.06 \\
(0.73,1.55)\end{array}$ & $\begin{array}{c}1.03 \\
(0.70,1.50)\end{array}$ & \\
\hline
\end{tabular}

Chemotherapy: antineoplastic agents

only (procedure codes 96199-00,

96200-00, 96201-00, 96202-00,

96203-00, 96204-00, 96205-00,

96206-00, 96207-00, 96208-00, 96209-00)

$n(\%)^{\text {a }}$ of women with diagnosis who had procedure

$\begin{array}{cccc}638 & 22(12.2 \%) & 234 & 382 \\ (20.0 \%) & & (15.5 \%) & (25.6 \%) \\ 624 & 57.4 & 49.9 & 9.5 \\ & (29.4,85.5) & (38.0,61.7) & (8.5,10.5)\end{array}$
procedure

Adjusted OR (95\% Cl) for procedure
$2330 \quad 1.00$
1.56

$(0.88,2.77)(1.25,4.04)$
- Age: OR 0.97 (95\% Cl 0.96, 0.98)

- Annual household income more than $\$ 70000$ (yes vs no): OR 1.37 $(95 \% \mathrm{Cl} 1.07,1.75)$

- Private health insurance with extras (yes vs no): OR 2.42 (95\% Cl 1.91, 3.05)

Average distance to health service (per $10 \mathrm{~km}$ ): OR 0.97 (95\% Cl 0.94 0.995)

- Cohort (rural nonfarm vs urban): OR $0.70(95 \% \mathrm{Cl} 0.55,0.88)$

\begin{tabular}{|c|c|c|c|c|c|}
\hline Colorectal cancer & 1401 & 80 & 711 & 610 & \\
\hline \multicolumn{6}{|l|}{$\begin{array}{l}\text { Colorectal surgery: colectomies, } \\
\text { resections and excisions (procedure } \\
\text { codes } 32000-00-32069-00 \\
32099-00-32108-00 \text { ) }\end{array}$} \\
\hline $\begin{array}{l}\mathrm{n}(\%)^{\mathrm{a}} \text { of women with diagnosis who } \\
\text { had procedure }\end{array}$ & $\begin{array}{c}1099 \\
(78.4 \%)\end{array}$ & $\begin{array}{c}61 \\
(76.3 \%)\end{array}$ & $\begin{array}{c}549 \\
(77.2 \%)\end{array}$ & $\begin{array}{c}489 \\
(80.2 \%)\end{array}$ & - $\quad$ Nil \\
\hline $\begin{array}{l}\text { Average distance }(\mathrm{km})(95 \% \mathrm{Cl}) \text { from } \\
\text { residence to health service for first } \\
\text { procedure }\end{array}$ & 1089 & $\begin{array}{l}92.9 \\
(63.4 \\
122.4)\end{array}$ & $\begin{array}{c}55.9 \\
(48.4,63.4)\end{array}$ & $\begin{array}{c}10.7 \\
(7.0,14.5)\end{array}$ & \\
\hline Adjusted OR (95\% Cl) for procedure & 1401 & 1.00 & $\begin{array}{c}1.06 \\
(0.61,1.82)\end{array}$ & $\begin{array}{c}1.26 \\
(0.73,2.19)\end{array}$ & \\
\hline
\end{tabular}


Table 3. Procedures performed on farm, rural nonfarm and urban women who had a breast or colorectal cancer diagnosis on any APDC admission record, 2006-2012 (continued)

\begin{tabular}{|c|c|c|c|c|c|}
\hline Procedures & Total & Farm & $\begin{array}{c}\text { Rural } \\
\text { nonfarm }\end{array}$ & Urban & Factors remaining in adjusted model \\
\hline \multicolumn{6}{|l|}{$\begin{array}{l}\text { Chemotherapy: antineoplastic agents } \\
\text { only (procedure codes 96199-00, } \\
96200-00,96201-00,96202-00 \text {, } \\
96203-00,96204-00,96205-00 \\
96206-00,96207-00,96208-00, \\
96209-00)\end{array}$} \\
\hline $\begin{array}{l}n(\%)^{a} \text { of women with diagnosis who } \\
\text { had procedure }\end{array}$ & $\begin{array}{c}167 \\
(11.9 \%)\end{array}$ & $\begin{array}{c}9 \\
(11.3 \%)\end{array}$ & $\begin{array}{c}63 \\
(8.9 \%)\end{array}$ & $\begin{array}{c}95 \\
(15.6 \%)\end{array}$ & \multirow{3}{*}{$\begin{array}{l}\text { - Age: OR } 0.95(95 \% \mathrm{Cl} 0.94,0.97) \\
\text { - Private health insurance with extras } \\
\text { (yes vs no): OR } 2.07 \text { (95\% Cl 1.35, } \\
\text { 3.16) } \\
\text { - } \quad \text { Annual household income more } \\
\text { than } \$ 70000 \text { (yes vs no) was a } \\
\text { nonsignificant confounder; OR } 1.16 \\
\text { (95\% Cl 0.68, 2.00)] } \\
\text { - Cohort (rural nonfarm vs urban): } \\
\text { OR } \mathbf{0 . 5 7}(\mathbf{9 5 \%} \mathbf{C l} \mathbf{0 . 3 7}, \mathbf{0 . 8 8})\end{array}$} \\
\hline $\begin{array}{l}\text { Average distance }(\mathrm{km})(95 \% \mathrm{Cl}) \text { from } \\
\text { residence to health service for first } \\
\text { procedure }\end{array}$ & 159 & $\begin{array}{l}71.1 \\
(20.3 \\
122.0)\end{array}$ & $\begin{array}{r}82.5 \\
(49.5 \\
115.5)\end{array}$ & $\begin{array}{c}15.8 \\
(2.9,28.6)\end{array}$ & \\
\hline Adjusted OR (95\% Cl) for procedure & 951 & 1.00 & $\begin{array}{c}0.84 \\
(0.35,2.01)\end{array}$ & $\begin{array}{c}1.47 \\
(0.62,3.49)\end{array}$ & \\
\hline
\end{tabular}

APDC = Admitted Patient Data Collection; $\mathrm{Cl}=$ confidence interval; $\mathrm{OR}=$ odds ratio Bold text $=$ odds ratio significant compared with referent resident group to 0.05 level

a Chi-square test of proportions between resident groups significant to 0.05 level

\section{Discussion}

Although farm-specific data about provision of prostate, breast and colorectal cancer therapies are unavailable in the Australian literature, results for the general rural population that includes farm residents are mixed. Some studies have reported comparable levels of cancerrelated surgery and chemotherapy in urban and rural cancer patients. ${ }^{13-16}$ Others have reported lower rates of prostatectomy, breast-conserving surgery, radiotherapy and chemotherapy in rural areas. ${ }^{2,14-18}$

Apart from socio-economic difficulties related to travel, the availability of specialist services can be an issue in rural areas. ${ }^{2}$ Limited access to specialist urologists was one reason suggested for lower rates of radical prostatectomy in rural areas in the $1990 \mathrm{~s}^{19}$, but farm and rural nonfarm men were not disadvantaged compared with urban men in our study. It is not known why farm men underwent more prostatectomies than rural nonfarm men, despite longer distances to treatment and similar prostate cancer risk in the two groups (reported elsewhere ${ }^{8}$ ). Urban men were more likely than either rural group to have chemotherapy and brachytherapy - a viable alternative to radical prostatectomy for early prostate cancer. However, these treatments are available in less than half of all Australian radiation oncology facilities. ${ }^{20}$

Rates of surgery for colorectal cancer were reportedly lower among rural residents than among urban residents in Queensland, but comparable in South Australia. ${ }^{13-16}$ In contrast, the proportion of surgical procedures for breast cancer was lower in rural women in Queensland and Western Australia than in urban counterparts. ${ }^{14,16.21}$ In the current study, the rate of chemotherapy administration was higher among urban people than rural nonfarm residents for colorectal cancer, plus higher than both rural groups for breast cancer. This is consistent with delayed or lower odds of chemotherapy for breast and colorectal cancer patients in rural NSW, reported previously. ${ }^{17-18}$ In contrast, studies from South Australia and Western Australia have reported similar levels of chemotherapy for breast and colorectal cancers in urban and rural patients. ${ }^{13-14}$ More centralised treatment models have developed in these states to service sparse rural populations, which contrasts with NSW and its sizeable regional populations. ${ }^{13}$

Findings for chemotherapy and radiotherapy in our study should be considered with some caution because of low case numbers, which affect confidence intervals and the potential significance of results. Validation studies have also found these procedures to be underreported in the APDC, with prostate cancer therapies less accurately recorded for nonurban cases. Potentially, the latter could lead to a positive bias towards higher odds of prostate cancer therapies among urban men. In contrast, surgical treatments and brachytherapies are usually well captured. ${ }^{22-23}$

Other limitations include the 45 and Up Study cohort not being completely representative of the NSW population, although linkage with population datasets has shown that such biases are limited. Also, the definition of 'farm resident' in this study was open to respondents' 
interpretation of 'farm'; therefore, characteristic exposures, practices and lifestyles of farm residents may be more varied. This could affect the representativeness of subgroups. However, misclassification errors are likely to be nondifferential and independent of other risk factors, leading to a null bias and underestimation of relationships between farm residence and cancer therapy.

\section{Conclusion}

Acknowledging limitations, this study found that cancerrelated surgical services were generally comparable between farm, rural nonfarm and urban residents for breast and colorectal cancers. Farm men were more likely to have a prostatectomy than rural nonfarm men, and urban men had greater use of brachytherapy than either rural group. Generally higher use of nonsurgical therapies by urban residents in this study (up to 2012), supports earlier reports of disadvantage in specialist cancer services in rural areas. ${ }^{2}$ In addition, the study suggests that this disadvantage is similar across farm and rural nonfarm resident groups.

Challenges for providing oncology services in rural areas include rural demographics; limitations in communications technology; difficulty in recruiting and retaining technically qualified personnel; and challenges in planning and coordinating surgical, pathology and oncology services. ${ }^{13,17,19}$ Ongoing development of the Rural Cancer Centres initiative, telemedicine and CanNET cancer networking services are likely to address ruralurban imbalances, and improve the accessibility, quality and coordination of rural cancer services. ${ }^{25}$

\section{Acknowledgements}

The authors thank the 45 and Up Study and partners. The 45 and Up Study is managed by the Sax Institute in collaboration with major partner Cancer Council NSW; and partners: the National Heart Foundation of Australia (NSW Division); NSW Ministry of Health; NSW Government Family \& Community Services - Ageing, Carers, and the Disability Council NSW; and the Australian Red Cross Blood Service. Thanks also to the many thousands of people participating in the 45 and Up Study.

\section{Peer review and provenance}

Externally peer reviewed, not commissioned.

\section{Competing interests}

None declared.

\section{Author contributions}

JD conceived and designed the analysis, reviewed the literature, analysed and interpreted the data and drafted and revised the manuscript. TL interpreted and critically reviewed the data and revised the manuscript. BA interpreted and critically reviewed the data and provided feedback on the manuscript. TD critically reviewed the methods and provided feedback on the manuscript. All authors read and approved the final manuscript.

\section{References}

1. Australian Institute of Health and Welfare. Cancer in Australia: an overview 2014. Canberra: AIHW; 2014 [cited 2017 Mar 10]. Available from: www. aihw.gov.au/getmedia/79c940b1-2438-45c8-99e2a4b593253ccd/18114.pdf.aspx?inline=true

2. Heathcote KE, Armstrong BK. Disparities in cancer outcomes in regional and rural Australia. Cancer Forum. 2007.31(2):70-4.

3. Australian Institute of Health and Welfare. Australian health expenditure by remoteness: a comparison of remote, regional and city health expenditure. Canberra: AlHW; 2011 [cited 2017 Mar 10]. Available from: www. aihw.gov.au/reports/health-welfare-expenditure/australianhealth-expenditure-by-remoteness-a-com/contents/tableof-contents

4 Australian Bureau of Statistics. Australian Statistical Geography Standard (ASGS) Remoteness Structure Australia. Canberra: ABS; 2011 [cited 2017 Mar 10]; [about 2 screens]. Available from: www.abs.gov.au/ ausstats/abs@.nsf/mf/1270.0.55.005

5. Australian Bureau of Statistics: 4102.0 Australian social trends. Australian farming and farmers. Canberra: ABS; 2012 [cited2017 Mar 10]; [about 10 screens]. Available from:www.abs.gov.au/AUSSTATS/abs@.nsf/Lookup/4102. OMain+Features10Dec+2012

6. Donham K, Thelin A. Agricultural medicine: rural occupational and environmental health, safety and prevention. 2nd ed. Wiley \& Sons; 2016. Donham K. Chapter 5, Cancer in agricultural populations; p. 181-203.

7. Fragar L, Depczynski J, Lower T. Mortality patterns of Australian male farmers and farm managers. Aust J Rural Health. 2011;19(4):179-84.

8. Depczynski J.A population-based examination of cancer in New South Wales farm residents compared to rural non-farm and urban residents [thesis]. Sydney: School of Public Health, University of Sydney; 2017.

9. The Sax Institute. Sydney: Sax Institute. 45 and Up Study [cited 2017 Mar 10]; [about 2 screens]. Available from: www.saxinstitute.org.au/our-work/45-up-study/

10. NSW Department of Health. Admitted Patient Data Collection - variable information. Unpublished notes to researchers. October 2013. 
11. Centre for Health Record Linkage. Sydney: NSW Health; 2018. How record linkage works: technical details; c2018 [cited 2017 Mar 10]; [about 1 page]. Available from: www.cherel.org.au/how-record-linkage-works/technicaldetails

12. Australian Bureau of Statistics. Household income and income distribution, Australia: 2005-06. Canberra: ABS; 2009 [cited 2017 Mar 10]. Available from: www.ausstats. abs.gov.au/Ausstats/subscriber.nsf/0/447BC40F0713F45 4CA25732A00258F43/\$File/65230_2005-06.pdf

13. Hocking C, Broadbridge VT, Karapetis C, Beeke C, Padbury R, Maddern GJ, et al. Equivalence of outcomes for rural and metropolitan patients with metastatic colorectal cancer in South Australia. Med J Aust. 2014;201(8):462-6.

14. Mitchell KJ, Fritschi L, Reid A, McEvoy SP, Ingram DM, Jamrozik K, et al. Rural-urban differences in the presentation, management and survival of breast cancer in Western Australia. Breast. 2006;15:769-76.

15. Craft P, Buckingham JM, Dahlstrom JE, Beckmann KR, Yanping Z, Stuart-Harris R, et al. Variation in the management of early breast cancer in rural and metropolitan centres: implications for the organisation of rural cancer services. Breast. 2010;19(5):396-401.

16. Tam R, Harden H, Zarate D, Colquist S, Walpole E. Patterns of cancer surgery in rural versus urban cancer patients. Paper presented at: Clinical Oncology Society of Australia Annual Scientific Meeting; 2013 Nov 12-14; Adelaide, South Australia.

17. Fox PN, Chatfield MD, Beith JM, Allison S, DellaFiorentina S, Fisher D, et al. Factors delaying chemotherapy for breast cancer in four urban and rural oncology units. ANZ J Surg. 2013;83(7-8):533-8.

18. Young JM, Leong DC, Armstrong K, O'Connell D, Armstrong BK, Spigelman AD, et al. Concordance with national guidelines for colorectal cancer care in New South Wales: a population-based patterns of care study. Med J Aust. 2007;186(6):292-5.
19. Baade P, Youlden D, Coory M, Gardiner R, Chambers S. Urban-rural differences in prostate cancer outcomes in Australia: what has changed? Med J Aust. 2011;194(6):293-6.

20. Radiation Oncology Tripartite Committee. Planning for the best: tripartite national strategic plan for radiation oncology 2012-2022. Sydney: Royal Australian and New Zealand College of Radiologists; 2012 [cited 2017 Mar 10]. Available from: www.ranzcr.com/college/ document-library/radiation-oncology-tripartite-nationalstrategic-plan-2012-2022

21. Green T, Zarate D, Colquist S, Harden H, Walpole E. Patterns of mastectomy for invasive breast cancer in Queensland. Clinical Oncology Society of Australia Annual Scientific Meeting; 2013 Nov 12-14; Adelaide, South Australia.

22. Goldsbury DE, Armstrong K, Simonella L, Armstrong BK, O'Connell DL. Using administrative health data to describe colorectal and lung cancer care in New South Wales, Australia: a validation study. BMC Health Serv Res. 2012;12(1):387.

23. Goldsbury DE, Smith DP, Armstrong BK, O'Connell DL. Using linked routinely collected health data to describe prostate cancer treatment in New South Wales, Australia: a validation study. BMC Health Serv Res. 2011;11(1):253.

24. Mealing NM, Banks E, Jorm LR, Steel DG, Clements MS, Rogers KD. Investigation of relative risk estimates from studies of the same population with contrasting response rates and designs. BMC Med Res Methodol. 2010;10:26.

25. Herron L. Improving cancer care for rural Australians. Report of the Clinical Oncological Society of Australia and the Medical Oncology Group of Australia. 10th National Rural Health Conference; 2009 May 17-20; Cairns, Australia [cited 2017 Mar 10]. Available from: www.ruralhealth.org.au/10thNRHC/10thnrhc.ruralhealth. org.au/papers/docs/Herron_Lisa_COSA_Report.pdf

\section{Copyright: (c) ()}

(C) 2018 Depczynski et al. This article is licensed under the Creative Commons Attribution-NonCommercial-ShareAlike 4.0 International Licence, which allows others to redistribute, adapt and share this work non-commercially provided they attribute the work and any adapted version of it is distributed under the same Creative Commons licence terms. See: www.creativecommons.org/licenses/by-nc-sa/4.0/ 